\title{
Clinical Characteristics of Heart Failure from Case Reports Presented at the Regional Meeting of the Japanese Society of Internal Medicine
}

\author{
Tomoyuki Kabutoya ${ }^{1}$, Hisahiko Sato ${ }^{2}$, Eiji Aramaki $^{3}$, Kazuomi Kario ${ }^{1}$ and Ryozo Nagai ${ }^{4}$
}

\begin{abstract}
:
Objective To examine case reports presented at the Regional Meeting of the Japanese Society of Internal Medicine in order to clarify the underlying disease and prognosis of heart failure, which is often caused by non-cardiovascular diseases.

Methods We examined 49,693 case reports from the Japanese Society of Internal Medicine database. A total of 2,893 reports were included after excluding 46,022 reports that did not include the term "heart failure" and 778 reports with no indications of symptoms of heart failure. We assessed each patient's basal disease, and according to the abstracts, we reported their prognosis as dead or alive.

Results Of the 2,893 reports included, $1,952(67.5 \%)$ and 941 (32.5\%) had cardiovascular and noncardiovascular diseases as the causes, respectively; these cases were attributed to 725 different diseases, 196 $(27.0 \%)$ and $529(73.0 \%)$ of which were cardiovascular and non-cardiovascular diseases, respectively. In addition, 91 different side effects were identified. The percentage of cases of heart failure-related mortality was significantly higher among the patients with non-cardiovascular diseases than in those with cardiovascular diseases $(17.8 \%$ vs. $10.8 \% ; \mathrm{p}<0.001)$. Of the diseases reported as causes of heart failure in more than 10 reports, pulmonary tumor thrombotic microangiopathy $(87 \%)$, multiple myeloma (50\%), and amyloidosis $(47 \%)$ accounted for the highest percentages of heart failure-related mortality.

Conclusion Because heart failure is often caused by non-cardiovascular diseases, a broad study of case reports on internal medicine is important for cardiologists.
\end{abstract}

Key words: heart failure, case report, internal medicine

(Intern Med 58: 2145-2150, 2019)

(DOI: 10.2169/internalmedicine.2583-18)

\section{Introduction}

Heart failure (HF), a cardiac disease, is an important concern owing to its high prevalence and associated mortality. There are more than 6.5 million adults with $\mathrm{HF}$ in the United States (1). In Japan, the number of patients with HF is expected to reach 1.2 million by 2020 (2).

$\mathrm{HF}$ is often caused by ischemic heart disease, hypertension, or valvular disease (3-5). However, many cases of HF are also caused by systemic diseases and malignant tumors.
Hence, patients with HF are likely to be examined by both cardiologists and non-cardiologists at hospitals or clinics. For example, there are various symptoms and causes of Takotsubo cardiomyopathy, and case reports have been published from different types of hospital and clinical departments. Thus, non-cardiologists must understand HF, and cardiologists must be aware of the underlying noncardiovascular diseases that can lead to HF.

Learning the differential diagnoses of HF will help both cardiologists and non-cardiologists. The studies conducted by Harrison (6), Goldman-Cecil (7), and Braunwald (8) pro-

\footnotetext{
${ }^{1}$ Division of Cardiovascular Medicine, Department of Medicine, Jichi Medical University School of Medicine, Japan, ${ }^{2}$ Precision Inc., Japan, ${ }^{3}$ Graduate School of Information Science, Nara Institute of Science and Technology, Japan and ${ }^{4}$ Jichi Medical University School of Medicine, Japan

Received: December 28, 2018; Accepted: March 24, 2019; Advance Publication by J-STAGE: June 7, 2019

Correspondence to Dr. Tomoyuki Kabutoya, kabu@jichi.ac.jp
} 


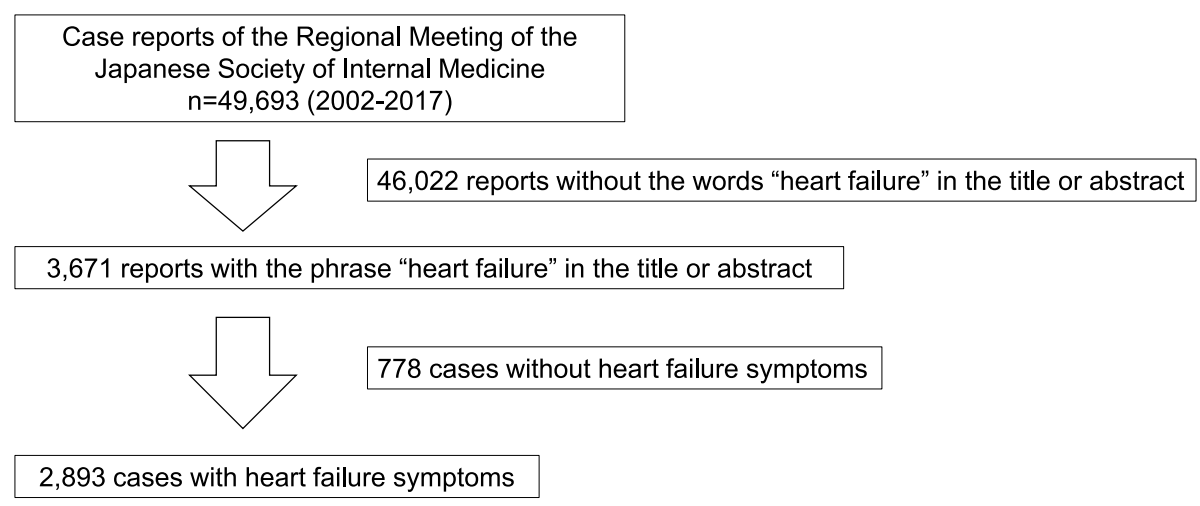

Figure 1. Flowchart of the search for case reports on heart failure.

vide a systematic understanding of HF. However, these studies cannot be expected to have completely described the diversity of HF cases; furthermore, frequent updates are difficult to carry out.

The Taiwan National Health Insurance Program is a nationwide initiative; its database contains detailed background characteristics of patients with HF (9). However, in the database, it is unclear what percentage of cases was compiled by non-cardiologists. Consequently, we sought to clarify the nature of the underlying diseases and HF-related fatalities by analyzing case reports presented at the Japanese Society of Internal Medicine. Of note, these reports originated from both cardiovascular and non-cardiovascular departments.

\section{Materials and Methods}

The Japanese Society of Internal Medicine holds regional meetings in Japan (e.g. in the Kanto region, Kinki region, etc.) nine times a year. Since 1946, 1,000 case reports have been presented at these meetings. Since 2002, reports presented at regional meetings across Japan have been published on the conference website, and the total number of reports in the conference database now exceeds 49,000.

Using an editing tool for abstracts (Shourei-Kun; https:// www.naika.or.jp/info/info_kensaku/), we examined 49,693 case reports from the Japanese Society of Internal Medicine database deposited from 2002 to 2017. We excluded 46,022 reports that did not include the phrase "heart failure" based on a simple text search. After peer-reviewing the abstracts, we also excluded 778 reports with no reported symptoms of HF. We also determined the basal disease, reporting department, and prognosis for the remaining 2,893 cases with HF (5.8\% of 49,693 case reports; Fig. 1). The prognosis was classified as dead or alive, according to the abstracts.

The cases originated across the following 10 department types: 1) cardiology, 2) allergy and rheumatology, 3) general internal medicine, 4) infectious disease, 5) hematology, 6) respiratory medicine, 7) gastroenterology, 8) neurology, 9) nephrology, and 10) endocrinology and metabolism. We further classified the cases into the following 18 categories: 1) cardiovascular disease, 2) congenital heart disease, 3) cardiac tumor, 4) connective tissue diseases, 5) infectious dis- eases, 6) hematological diseases, 7) respiratory diseases, 8) gastrointestinal hepatic, biliary and pancreatic diseases, 9) neurological diseases, 10) kidney diseases, 11) endocrine diseases, 12) malignant tumors, 13) hematological malignancies, 14) inflammatory disorders, 15) systemic disorders, 16) genetic disorders, 17) side effects, and 18) others. Supplementary material 1 presents the cases classified under connective tissue diseases, systemic disorders, and genetic disorders. We subsequently combined categories 1)-3) as cardiovascular disease cases and categories 4)-18) as noncardiovascular disease cases.

We used an unpaired $t$-test and analysis of variance (ANOVA) to evaluate and compare, respectively, the percentage of fatal HF cases among the case reports originating from the cardiovascular and non-cardiovascular departments. We analyzed all data using the Statistical Package for the Social Sciences software program (SPSS v.16.0; Chicago, USA). $\mathrm{p}<0.05$ was considered statistically significant.

\section{Results}

Of the 2,893 total case reports on HF, 1,952 (67.5\%) and $941(32.5 \%)$ originated from cardiovascular and noncardiovascular departments, respectively (Table 1). The etiology was cardiovascular disease in 1,181 cases $(40.8 \%)$ and non-cardiovascular disease in 1,712 (59.2\%).

Table 2 presents the underlying diseases for more than 10 cases. Amyloidosis and sarcoidosis were the major underlying non-cardiovascular diseases, whereas ischemic heart disease, dilated cardiomyopathy, Takotsubo cardiomyopathy, and infective endocarditis were the major underlying cardiovascular diseases. Fig. 2 presents the underlying diseases (total 30) and the originating department of each case of Takotsubo cardiomyopathy; although the reports were mostly from cardiology departments $(68.1 \%)$, the major underlying causes were endocrine disease (20.2\%), infectious disease $(13.0 \%)$, respiratory disease $(7.2 \%)$, and nervous disease $(7.2 \%)$.

In all, 725 diseases were identified, including 196 (27.0\%) types of cardiovascular disease and 529 (73.0\%) types of non-cardiovascular diseases. In addition, 91 different side effects were identified (Fig. 3). Fig. 4 shows the 
Table 1. Case Reports of Heart Failure Classified by Department of Origin.

\begin{tabular}{lccc}
\hline \multicolumn{1}{c}{ Department } & Number of reports & $\begin{array}{l}\text { Case reports with the } \\
\text { phrase "heart failure" } \\
\text { in the title or abstract }\end{array}$ & Cases of heart failure \\
\hline Cardiology & 7,761 & 2,285 & $1,952(67.5)$ \\
Allergy and rheumatology & 3,983 & 132 & $111(3.8)$ \\
General internal medicine & 423 & 16 & $13(0.4)$ \\
Infectious diseases & 3,952 & 114 & $64(2.2)$ \\
Hematology & 4,728 & 170 & $126(4.4)$ \\
Respiratory medicine & 5,685 & 201 & $144(5.0)$ \\
Gastroenterology & 8,419 & 160 & $69(2.4)$ \\
Neurology & 4,634 & 89 & $50(1.7)$ \\
Nephrology & 3,535 & 203 & $132(4.6)$ \\
Endocrinology and metabolism & 6,573 & 301 & $232(8.0)$ \\
Total & 49,693 & 3,671 & $2,893(100)$ \\
\hline
\end{tabular}

Data is shown as number or percentage of originating department in cases of heart failure.

Table 2. Underlying Diseases Implicated in More than 10 Cases.

\begin{tabular}{lcll}
\hline \multicolumn{1}{c}{ Disease } & Number & & \\
\hline Amyloidosis & 155 & Hypertrophic obstructive cardiomyopathy & 19 \\
Ischemic heart disease & 112 & Acromegaly & 19 \\
Infectious endocarditis & 89 & Hyperthyroidism & 17 \\
Dilated cardiomyopathy & 71 & Multiple myeloma & 16 \\
Takotsubo myopathy & 69 & Vasospastic angina & 16 \\
Sarcoidosis & 66 & Acute myocarditis & 16 \\
Acute myocardial infarction & 65 & Fulminant myocarditis & 16 \\
Vitamin B1 deficiency & 44 & Hypertensive heart disease & 16 \\
Thyroid crisis & 43 & Polymyositis & 15 \\
Systemic lupus erythematosus & 35 & Pulmonary tumor thrombotic microangiopathy & 15 \\
Graves' disease & 35 & Malignant hypertension & 14 \\
Aortic valve stenosis & 34 & Anthracycline-associated & 13 \\
Eosinophilic myocarditis & 34 & Pulmonary hypertension & 12 \\
Atrial fibrillation & 32 & Trastuzumab-associated & 12 \\
Constrictive pericarditis & 31 & Corrected transposition of the great arteries & 12 \\
Non-compaction & 31 & Sjögren's syndrome & 11 \\
Peripartum cardiomyopathy & 30 & Rheumatoid arthritis & 11 \\
Hyperthyroidism & 30 & Tuberculous pericarditis & 11 \\
Malignant lymphoma & 29 & Cushing's syndrome & 11 \\
Mitochondrial disease & 28 & Lung cancer & 11 \\
Systemic scleroderma & 26 & Crow-Fukase's syndrome & 11 \\
Aortitis syndrome & 24 & Acute coronary syndrome & 10 \\
Obesity hypoventilation syndrome & 23 & After mitral valve replacement & 10 \\
Mitral regurgitation & 22 & Dermatomyositis & 10 \\
Pheochromocytoma & 20 & Sleep apnea syndrome & 10 \\
Renal artery stenosis & 19 & Fabry disease & 10 \\
\hline
\end{tabular}

percentage of fatal HF cases according to the originating department. The percentages of fatal HF cases were high among the reports originating from the hematology $(27.0 \%)$, respiratory medicine $(30.6 \%)$, and gastroenterology $(27.7 \%)$ departments. The percentage of fatal HF cases was significantly higher among reports originating from noncardiovascular departments than among those originating from cardiovascular departments $(17.8 \%$ vs. $10.8 \%$, respectively; $\mathrm{p}<0.001)$. The diseases implicated in more than 10 cases were pulmonary tumor thrombotic microangiopathy (PTTM; 87\%), multiple myeloma (50\%), and amyloidosis (47\%). These diseases accounted for the highest percentages of HF-related mortality. The number of reported PTTM cases (by year) is presented in Table 3. Although PTTM is associated with a high mortality rate, surviving cases were reported in 2012 and 2017. 


\section{(A)}

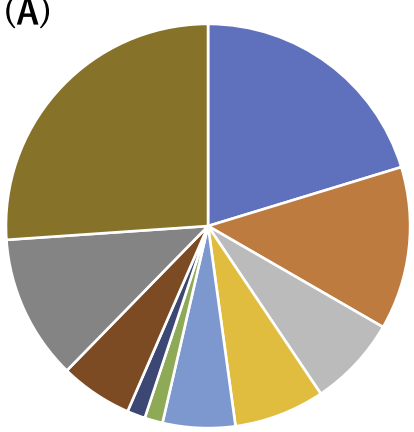

- Endocrine disease $20.3 \%$

- Infectious disease $13.0 \%$

- Respiratory disease $7.2 \%$

- Nervous disease $7.2 \%$

- Cardiovascular disease 5.8\%

- Hematological disease $1.4 \%$

- Kidney disease $1.4 \%$

- Stress $5.8 \%$

- Others $11.6 \%$

- Unknown $26.1 \%$

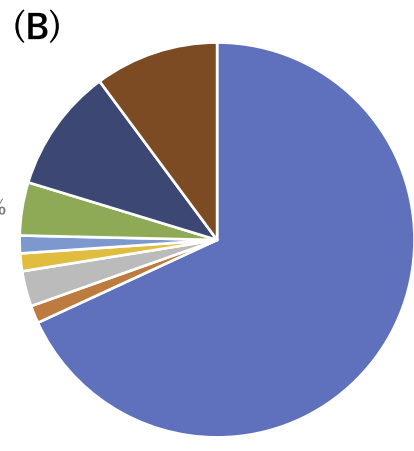

- Cardiology $68.1 \%$

Allergy and rheumatology $1.4 \%$

Infectious disease $2.9 \%$

Respiratory medicine $1.4 \%$

Gastroenterology $1.4 \%$

Neurology $4.3 \%$

Nephrology $10.1 \%$

Endocrinology and metabolis $10.1 \%$

Endocrine disease 14: Electrolyte abnormalities 4, thyroid gland malfunction 4, adrenal disease 2, hypoglycemia 1, hyperglycemia 1, dehydration 1, lactic acidosis 1

Onfectious disease 9: pneumonia 3, sepsis 3 , influenza 1, enterocolitis 1, unknown 1

Respiratory disease 5: bronchial asthma 3 , hemoptysis 1 , type 2 chronic respiratory failure 1

ONervous disease 5: cerebral infarction 2, cerebral bleeding 1, subarachnoid Hemorrhage 1, metastatic brain tumor 1

- Cardiovascular disease 4: Adams-Stokes attack 1, paroxysmal atrial fibrillation 1, long QT syndrome 1, renal artery stenosis 1

- Hematological disease 1: thrombotic thrombocytopenic purpura 1

Kidney disease 1: acute renal failure 1

OStress 4

Others 8: after superficial temporal artery biopsy 1 , fall 1 , Caesarean section 1, antibiotic allergy 1 , rhabdomyolysis 1 , MPO-ANCA positive vasculitis 1 , anorexia nervosa 1 , femoral neck fracture 1

Unknown 18

Figure 2. Causes of diseases (A) and the originating departments (B) for the case reports on Takotsubo cardiomyopathy.

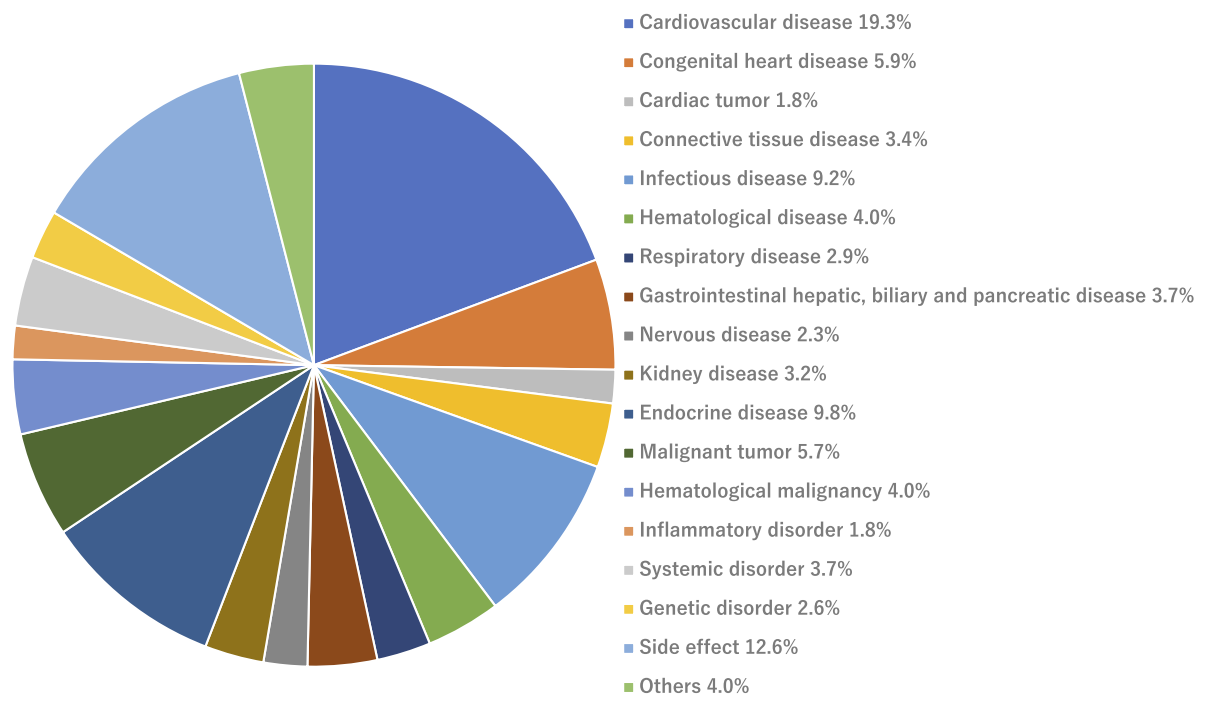

Figure 3. The number of diseases identified in $\mathbf{1 8}$ categories.

\section{Discussion}

HF case reports accounted for $5.8 \%$ of all internal medicine case reports. Of the 2,893 cases of $\mathrm{HF}, 40 \%$ were caused by cardiovascular diseases; however, the underlying cardiovascular disease was identified in only $20 \%$ of all cases.

We assessed all cases of HF in our conference database to determine the different underlying diseases. It has recently become possible to perform the differential diagnosis of $\mathrm{HF}$ using information available on the Internet (e.g. websites like UpToDate.com.) and in medical textbooks (e.g. those authored by Harrison, Goldman-Cecil, Braunwald, etc.) (6-8). The leading textbooks are growing thicker each year with the increase in knowledge concerning these diseases, making the Internet increasingly useful for its convenience and ease of access. However, the information available on the Internet is not always up-to-date due to delays in publication; furthermore, racial differences in the available sources may exist. In Japan, Takotsubo cardiomyopathy is a cause of HF, and in recent years, this disease has been frequently reported and garnered substantial attention (10). However, because there are several causes of Takotsubo cardiomyopathy, the relevant reports have originated from different types of hospitals and clinical departments. In the present study, we identified 725 separate disease designations. It is thus impractical to describe all of the potential underly- 


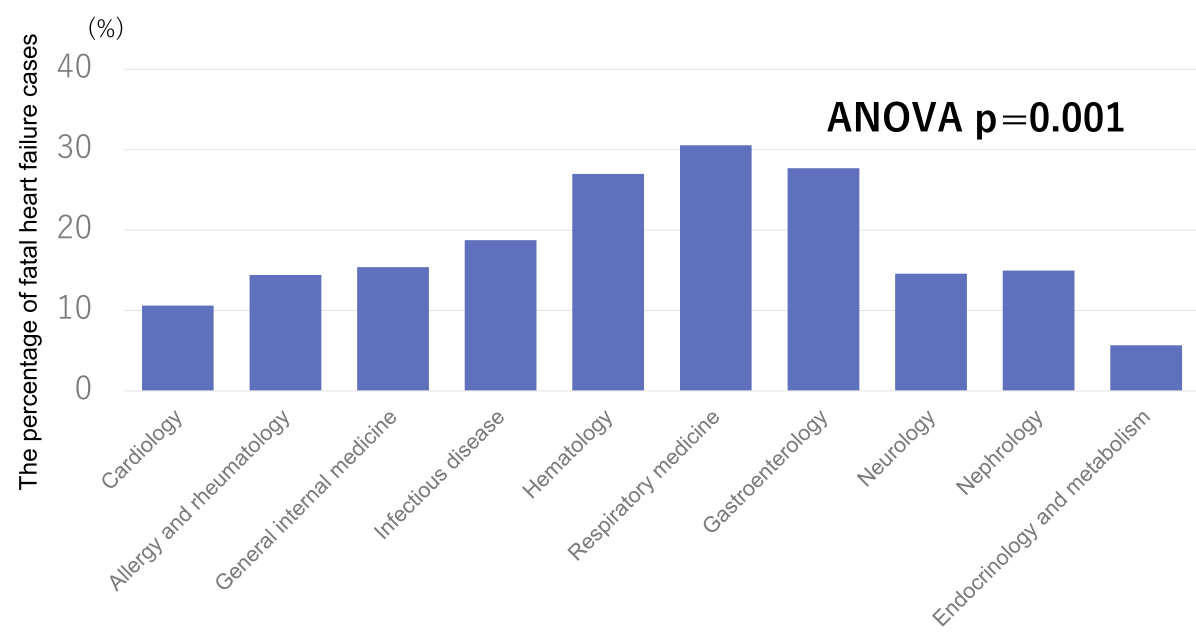

Figure 4. The percentage of fatal heart failure cases.

Table 3. Reported Number of Pulmonary Tumor Thrombotic Microangiopathies by Year.

\begin{tabular}{ccc}
\hline Year & Alive report & Death report \\
\hline 2006 & 0 & 1 \\
2007 & 0 & 0 \\
2008 & 0 & 0 \\
2009 & 0 & 0 \\
2010 & 0 & 1 \\
2011 & 0 & 2 \\
2012 & 1 & 2 \\
2013 & 0 & 1 \\
2014 & 0 & 1 \\
2015 & 0 & 3 \\
2016 & 0 & 1 \\
2017 & 1 & 1 \\
\hline
\end{tabular}

ing diseases in a single textbook. However, using case reports from the Regional Meeting of the Japanese Society of Internal Medicine database, we were able to investigate the details of the underlying diseases in recent cases of HF, including Takotsubo cardiomyopathy. The side effects of various therapeutic drugs could also be quickly checked using this database.

We found that a high percentage of HF-related mortality was attributable to PTTM, multiple myeloma, and amyloidosis. The differential diagnosis is therefore extremely important for clinicians. Both amyloidosis and sarcoidosis are systemic diseases. When non-cardiologists diagnose a systemic disease, they should pay attention to HF. Cardiologists should be able to properly diagnose and treat HF associated with systemic diseases. In the present study, PTTM was the underlying cause of HF-related mortality in many early autopsy reports; however, surviving cases have recently been reported. The first report of a patient with PTTM was published in 1990 (11), and the first such report in Japan was published in 1995 (12). The recently increasing PTTM incidence may be due to an improved disease understanding.
Further reports of detailed autopsy investigations in HF may help improve the quality of the diagnosis, treatment, and prognosis for rare diseases that cause HF.

In the present study, we attempted to elucidate the different etiologies of HF based on a large database of case reports. Of note, case reports (by their nature) often depict special or rare cases, so the disease frequencies and severities determined purely from case reports may differ from the actual status. However, the enumeration of many differential diagnoses is advantageous for the accurate diagnosis of HF etiologies and for identifying the underlying diseases most commonly associated with high mortality. In the future, by continued the annual updating of case reports, the number of diseases associated with HF will continue to increase, and the expanding database will improve our ability to differentially diagnose patients and determine the prognosis of HF.

\section{Conclusion}

Many cases of HF are caused by non-cardiovascular diseases. To improve our ability to clinically diagnose HF, it is necessary to examine the underlying non-cardiovascular diseases of HF. A broad study of case reports on internal medicine is important for cardiologists.

\section{Author's disclosure of potential Conflicts of Interest (COI).}

Hisahiko Sato: Stock ownership or options, Precision. Kazuomi Kario: Honoraria, Daiichi Sankyo, Omron Healthcare and Takeda; Research funding, A\&D, Bayer Yakuhin, Boehringer Ingelheim, Daiichi Sankyo, EA Pharma, Fukuda Denshi, Medtronic, Mitsubishi Tanabe Pharma Corporation, Mochida Pharmaceutical, Omron Healthcare, Otsuka, Pfizer, Takeda and Teijin Pharma.

\section{Financial Support}

This study was funded by the ImPACT program of the Council for Science, Technology and Innovation (Cabinet Office, Government of Japan). 


\section{References}

1. Benjamin EJ, Virani SS, Callaway CW, et al. Heart disease and stroke statistics-2018 update: a report from the American Heart Association. Circulation 137: e67-e492, 2018.

2. Okura Y, Ramadan MM, Ohno Y, et al. Impending epidemic: future projection of heart failure in Japan to the year 2055. Circ J 72: 489-491, 2008.

3. Tsutsui $H$, Tsuchihashi-Makaya M, Kinugawa S, et al. JCARECARD Investigators. Clinical characteristics and outcome of hospitalized patients with heart failure in Japan. Circ J 70: 16171623,2006

4. Shiba N, Watanabe J, Shinozaki T, et al. CHART investigators. Analysis of chronic heart failure registry in the Tohoku district: third year follow-up. Circ J 68: 427-434, 2004.

5. Shiba N, Nochioka K, Miura M, et al. CHART-2 investigators. Trend of westernization of etiology and clinical characteristics of heart failure patients in Japan-first report from the CHART-2 study. Circ J 75: 823-833, 2011.

6. Jameson JL, Fauci AS, Kasper DL, Hauser SL, Longo DL, Loscalzo J. Harrison's Principles of Internal Medicine. 20th ed. McGraw-Hill, New York, 2018.
7. Goldman L, Schafer AI. Goldman-Cecil Medicine. 25th ed. Elsevier Saunders, Philadelphia, PA, 2016.

8. Zipes DP, Libby P, Bonow RO, Mann DL, Tomaselli GF. Braunwald's Heart Disease: A Textbook of Cardiovascular Medicine. 11th ed. Elsevier Saunders, Philadelphia, PA, 2018.

9. Lin TY, Chen CY, Huang YB. Evaluating the effectiveness of different beta-adrenoceptor blockers in heart failure patients. Int $\mathbf{J}$ Cardiol 230: 378-383, 2017.

10. Medina de Chazal H, Del Buono MG, Keyser-Marcus L, et al. Stress cardiomyopathy diagnosis and treatment: JACC state-of-theart review. J Am Coll Cardiol 72: 1955-1971, 2018.

11. von Herbay A, Illes A, Waldherr R, Otto HF. Pulmonary tumor thrombotic microangiopathy with pulmonary hypertension. Cancer 66: 587-592, 1990.

12. Sato Y, Marutsuka K, Asada Y, Yamada M, Setoguchi T, Sumiyoshi A. Pulmonary tumor thrombotic microangiopathy. Pathol Int 45: 436-440, 1995.

The Internal Medicine is an Open Access journal distributed under the Creative Commons Attribution-NonCommercial-NoDerivatives 4.0 International License. To view the details of this license, please visit (https://creativecommons.org/licenses/ by-nc-nd/4.0/).

(C) 2019 The Japanese Society of Internal Medicine Intern Med 58: 2145-2150, 2019 